\title{
Migración internacional de retorno de Estados Unidos a México en un contexto de crisis económica y política
}

\author{
Merari Stephanie Montoya-Ortiz* \\ José Aurelio Granados-Alcantar* \\ José Alfredo Jauregui-Díaz** \\ *Universidad Autónoma del Estado de Hidalgo, México \\ **Universidad Autónoma de Nuevo León, México
}

Resumen

Este artículo analiza la migración reciente de retorno de Estados Unidos a México durante el periodo de 2001 a 2014,cohorte temporal donde la proliferación de leyes estatales anti-inmigrantes en Estados Unidos resultó en un incremento sin precedentes de las expulsiones ocurridas desde el interior del territorio estadounidense. Así mismo, la crisis económica estadunidense, que inició en 2007 y alcanzó su punto álgido en 2008, se tradujo en un incremento de retorno a México, lo cual se refleja en la información de la Encuesta Nacional de la Dinámica Demográfica (ENADID), la migración de retorno fue de 490 mil en el periodo 2004-2009 periodo que comprende los años más fuertes de la crisis económica y luego, nuevamente, se contrajo a 260 mil para 2009-2014; mientras que la migración definitiva o permanente a la Unión Americana se mantuvo en alrededor del millón de personas en esos periodos (Ramírez y Aguado, 2013: 179). Además la reciente crisis migratoria y humanitaria en 2014 de los menores migrantes no acompañados que alcanzó la cifra histórica de más de 61 mil. Dadas estas circunstancias, el objetivo de la investigación es analizar la migración internacional de retorno de Estados Unidos a México para el periodo comprendido entre 2004 y 2014 el cual se dio bajo un escenario de crisis económica y política.

Palabras clave: Migración de retorno, leyes anti-inmigrantes, crisis económica.

Abstract

Return migration from the United States to Mexico in a moment of economic and political crisis

This paper examines the recent migration of return from the United States to Mexico during the period 2004 to 2014, a temporary cohort where proliferation of anti-immigrant state laws in the United States of America, such as the Sensenbrenner Act approved in December 2005 (Borderprotection, Antiterrorism and Illegal Immigration Control Act) and the Arizona SB 1070 Act passed in 2009, both aimed especially at Mexicans, resulting in an unprecedented increase in expulsions from within the United States. Likewise, the US economic crisis that began in 2007 and reached its peak in 2008 resulted in an increase in return to Mexico, that for 2004-2009 report 460 thousand migrants that come back to Mexico form USA and the most recent migratory crisis in 2014 of unaccompanied migrant minors, which achieved 61 thousand cases.

Key words: return and removals, anti-immigrant laws, economic crisis. 


\section{AnteCedentes de La MigRaCión de Retorno de Estados Unidos a México}

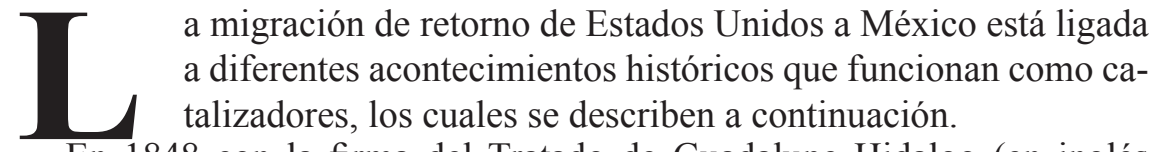

En 1848 con la firma del Tratado de Guadalupe Hidalgo (en inglés Treaty of Guadalupe Hidalgo), llamado también Tratado de Paz, Amistad, Límites y Arreglo Definitivo entre México y Estados Unidos, se pone fin a la guerra de intervención estadunidense cediendo más de la mitad del territorio mexicano, que comprende la totalidad de lo que hoy son los estados de California, Nevada, Utah, Nuevo México y Texas, y partes de Arizona, Colorado, Wyoming, Kansas y Oklahoma. Alrededor de $100 \mathrm{mil}$ ciudadanos mexicanos vivían en el terreno cedido por México, por ello, el gobierno de nuestro país publicó un Decreto el 19 de agosto de 1848 en el cual ofrecía que a todo aquel mexicano que lo solicitara, se le trasladaría a territorio nacional por cuenta del erario público y recibiría una dotación de tierras (Granados y Pizarro, 2013). Se calcula que cerca de 25 por ciento de la población de esos territorios regresó a México (Verduzco, 1995).

Una vez que se normalizaron las relaciones entre México y Estados Unidos se vivió una etapa de tránsito regular y cotidiano, sin la existencia de una legislación en materia de inmigración, sin embargo,

- Con la crisis de 1929 se incrementó el desempleo de manera estrepitosa en Estados Unidos, alcanzando tasas que sobrepasaron 20 por ciento, razón por la que el gobierno estadunidense hizo lo posible por preservar los puestos de trabajos a sus connacionales sacrificando a los inmigrantes (Sánchez, 2014). Douglas Massey llama a esta época la "era de las deportaciones", de 1929 a 1939, 469 mil ciudadanos mexicanos fueron invitados o forzados a salir de Estados Unidos, muchos de ellos acompañados por sus hijos, que eran ciudadanos americanos (Massey et al., 2009).

- Con el fin del programa bracero, en 1964, da inicio la inmigración no documentada y la circularidad migratoria. Se estima que entre 1965 y 1986, 85 por ciento de los migrantes indocumentados mexicanos que iban a Estados Unidos, regresaban de manera voluntaria o involuntaria (Massey et al., 2009). En 1965 se promulgó la ley Immigration and Nacionality Act, que por primera vez imponía limitaciones numéricas 
a la inmigración mexicana, política que se hará cada vez más restrictiva con las sucesivas enmiendas (Massey et al., 2009).

- Los atentados terroristas a las torres gemelas el 11 de septiembre de 2001, irrumpieron inesperadamente, modificando la dinámica migratoria; la migración se criminaliza y los inmigrantes son vistos como potenciales terroristas. Miles de mexicanos regresaron de manera forzosa y como consecuencia de una psicosis colectiva por temor a que ocurrieran más atentados.

- Durante 2001, el Instituto Nacional de Inmigración de México (INM) calcula que regresaron poco más de 350 mil mexicanos y la Encuesta sobre Migración en la Frontera Norte (EMIF Norte) captó 1.7 millones de eventos de retorno a México.

- La última oleada de migrantes de retorno registrados a la fecha es derivada de la crisis económica de 2007, la cual mermó las oportunidades de trabajo y desarrollo para los inmigrantes, se traduce en un incremento del flujo migratorio de retorno entre 2007 y 2009.

\section{OBJETIVO, METODOLOGÍA Y FUENTES DE INFORMACIÓN}

El objetivo central de este artículo es conocer la migración de retorno reciente que se ha visto impactada por las leyes antiinmigrantes aprobadas a partir de 2005, la crisis económica que inició en 2007 y la crisis humanitaria a raíz del incrementó de menores migrantes no acompañados en el año 2014. Las ENADID 2009 y 2014 dan cuenta de lo ocurrido a partir de dichos eventos, ya que, en conjunto, abarcan los años comprendidos entre 2004 y 2014, dando visibilidad al comportamiento de la migración de retorno en un periodo coyuntural. Complementándose por información publicada por la oficina de Immigration and Customs Enforcement (ICE), por su nombre en inglés, o Agencia de Aduana y Protección Fronteriza de Estados Unidos (CBP), que tiene información oficial del gobierno estadounidense referente a las remociones, retorno y menores migrantes no acompañados.

También existen diversas fuentes de información, tanto mexicanas como estadounidenses, que dan cuenta de la migración de retorno; entre las que se encuentran los Censo de Población y Vivienda de 2000 y 2010, el Conteo de Población y Vivienda de 1995 (CPV95), el Módulo de Migración Internacional de la Encuesta Nacional de Empleo de 2002 (ENE 2002), la Encuesta sobre Migración en la Frontera Norte de México, que se realiza anualmente desde 1999 (EMIF NORTE ), la Encuesta Nacional de Empleo Urbano, a partir de la edición de 2005 (ENOE) y la Encuesta 
Intercensal 2015, además de fuentes estadounidenses como las publicadas poe el Pew Research Center (PEW) e investigaciones periodísticas.

Se ha seleccionado como fuente principal de información para el procesamiento y análisis de datos de la presente investigación la ENADID (2009 y 2014), pues es la que ofrece una mayor cobertura de datos y detalles de información sobre migrantes internacionales de retorno, con una métrica basada en la persona. En cambio, la EMIF Norte, a pesar de ser una fuente especializada en migración internacional en la frontera México-Estados Unidos mide los eventos ocurridos; es decir, se enfoca en los flujos migratorios y no en las personas, por lo que un individuo puede ser captado en más de una ocasión durante la aplicación de la encuesta, por lo tanto, se encontró que la ENADID es la fuente óptima para el análisis de este tema. Del resto de las fuentes de información, se utilizan datos puntuales para complementar o respaldar el procesamiento de datos obtenida a partir de la ENADID. La ENADID 2009 y 2014 recaba información sobre los movimientos recientes de la migración internacional de retorno, es decir, nos permite acceder a una fuente de información que analiza los movimientos actuales sobre el fenómeno, y, por otro lado, es la fuente puntual que mide las causas de retorno, no por evento, como ocurre con la EMIF.

Es pertinente aclarar que una de las limitantes de la ENADID es que mide únicamente la migración reciente de retorno, pues pregunta por el lugar de residencia hace cinco años, por lo que se pierde el histórico de aquellos cuyo retorno es mayor de cinco años. Al considerar la ENADID 2009 y 2014 se abarcarán diez años, de 2004 a 2014, pero se da invisibilidad a aquellos cuyo retorno antes de 2004 sea mayor a los diez años, siendo una desventaja de la fuente seleccionada.

Para tener un panorama completo entre lo reportado por fuentes mexicanas y lo reportado por fuentes estadounidenses se incluyen cifras del ICE, que anualmente publica cifras de retornos y remociones (deportaciones), donde se pueden encontrar datos específicos de inmigrantes mexicanos, es decir, cuenta con información por nacionalidad.

A partir de la información de la ENADID 2009 y 2014, así como de CBP, se elaboraron tablas y gráficas con el fin de realizar un análisis a partir de la estadística descriptiva, que de cuenta de las variaciones y comportamiento de la migración de retorno, visualizando las tendencias existentes; además de un estudio de fuentes documentales que permitan profundizar el estudio y contar con el respaldo de otras investigaciones y reportajes al respecto.

Así mismo se incluye un análisis espacial, generado a partir de diversas fuentes periodísticas, que muestra la composición de Estados Unidos en función de la posición pro-migrante y anti-inmigrante, con la finalidad de 
entender la manera en que influyen estas posturas políticas en la migración de retorno. El mapeo se construyó asignando valor de " 0 " a aquellos estados que abiertamente han declarado tener una política anti-inmigrante, $\mathrm{y}$ valor de "1" a los de política pro-migrante, generando la base de datos a partir de la cual fue construido el mapeo elaborado con el software libre "Philcarto".

\section{MigRaCión de RETORNo EN UN CONTEXTO DE CRISIS: 2001-2014}

Algunos factores fungen como catalizadores del retorno de mexicanos, por ejemplo, falta de adaptabilidad, problemas culturales o el retorno forzoso por deportación, pero más allá de cuestiones particulares, se pueden identificar acontecimientos puntuales que incentivaron el regreso de los mexicanos; tal es el caso del atentado terrorista de 2001, ya que después de este suceso, el gobierno de Estados Unidos incrementó sus medidas de seguridad, entre las cuales se encuentra proteger sus fronteras. Por tanto, las políticas migratorias se volvieron foco de atención, lo cual pudo haber motivado el retorno de mexicanos. Es así como repentinamente el tema de la seguridad se convirtió en un factor decisivo de la agenda política de Estados Unidos, y la migración no quedó exenta. Por otro lado, la gente entró en una especie de psicosis colectiva, ya que temía por su vida y la de sus familias, lo cual provocó un aumento en el retorno de migrantes a México (Herrera et al., 2012).

Adicional al atentado terrorista de 2001, encontramos que tres acontecimientos coyunturales que han revitalizado las investigaciones sobre el retorno y sus consecuencias en las comunidades en México: en primera instancia, la reciente crisis económica de Estados Unidos en 2008, que afectó negativamente las oportunidades de empleo en los mercados de trabajo donde tradicionalmente laboraban los inmigrantes mexicanos en aquel país. La proliferación de leyes estatales anti-inmigrantes en Estados Unidos nos lleva al segundo acontecimiento, como la Ley Sensenbrenner aprobada en diciembre de 2005 (Borderprotection, Antiterrorism and Illegal Immigration Control Act), y en tercer, lugar la Ley Arizona SB 1070 aprobada en 2009, las cuales estaban dirigidas especialmente hacia los mexicanos lo que resultó en un incremento sin precedentes de las expulsiones ocurridas desde el interior del territorio estadounidense que ha aquejado a residentes y trabajadores mexicanos "no autorizados" (Anguiano et al., 2013), lo que nos lleva a deducir que hay un proceso de retorno y está presente en todo el país (Granados y Pizarro, 2013). Cabe señalar que la migración de retorno suele sobredimensionarse, porque en tiempos de 
crisis, el flujo de arribo se paraliza y pareciera que todos los inmigrantes están regresando a su país de origen, percepción que es errónea.

Por ende, la migración de retorno hoy en día implica un reto social, político y económico, ante el cual todos los actores - políticos, gobierno, migrantes y sociedad - juegan un rol clave, ya que se trata de un fenómeno que implica una oleada de cambios sociales, en particular la forma en que se ha venido dando durante la última década, que puede ser analizada desde diferentes matices, como grupos étnicos, regiones geográficas, entre otros. No obstante, como se ha establecido anteriormente, este proyecto se limita a analizar cómo se dan los flujos migratorios de retorno y las características sociodemográficas de los retornados, como son el género, grupos de edad, escolaridad, estrato, entre otros. En cuanto a cifras, tenemos que la Encuesta sobre Migración en la Frontera Norte de México, 2005 (COLEF, 2005) registró 708927 eventos procedentes de México con destino a Estados Unidos; en contraparte, hay 816907 migrantes procedentes de aquel país que regresan a México; es decir, por cada evento que cruza a Estados Unidos se registraron 1.15 eventos de retorno en 2005. Por otro lado, según datos de la Encuesta Nacional de la Dinámica Demográfica (ENADID), la migración circular quinquenal se redujo en casi la mitad, al pasar de 875 mil personas entre 1987 y 1992 a 490 mil en el periodo 20042009, y nuevamente se contrajo a 260 mil para 2009-2014; mientras que la migración definitiva o permanente a la Unión Americana se mantuvo en alrededor del millón de personas en esos periodos (Ramírez y Aguado, 2013: 179).

Wong, Resano y Martínez (2006), encontraron que la tendencia migratoria de retorno sigue un patrón similar al del volumen total del flujo migratorio, por lo que se observó un incremento de 300 mil personas en el periodo 1992-1997 con respecto a los migrantes de retorno para el quinquenio 1990-1995, pero al comparar el número de estos migrantes entre los quinquenios 1995-2000 y 1992-1997, se observa un decremento de aproximadamente 440 mil.

Los programas que atienden a este sector de la sociedad deben de cobrar importancia, pues el volumen de retornados en el siglo XXI es significativo, existen datos, como el del estudio divulgado en 2012 por el Centro Hispano Pew, en Washington, que dio a conocer que la oleada de $12 \mathrm{mi}-$ llones de mexicanos que emigró a Estados Unidos en los últimos 40 años registra una tasa de migración neta cero. El Pew atribuyó esa tendencia a factores como un débil mercado laboral e inmobiliario en Estados Unidos, más seguridad fronteriza y aumento en la cifra de deportaciones, así como 
a una caída del crecimiento demográfico en México y nuevas condiciones en la economía mexicana.

Es claro que la migración de retorno responde a una serie compleja de factores estructurales, políticos y sociales en ambos lados de la frontera, pero cuyo resultado es la reincorporación de migrantes a sus hogares, a sus comunidades y a sus regiones; estos son emigrantes retornados que requieren de servicios, que demandan empleos y que, traigan o no recursos monetarios o de capital humano, el hecho es que, con justo derecho, presionan sobre los recursos disponibles localmente (López, 2012).

Ahora bien, es importante tomar en cuenta que, una vez que el migrante retorna, se enfrenta a un proceso de toma de decisiones respecto a cómo será su desplazamiento al interior de México, para lo cual puede o no tomar en cuenta el bagaje de experiencias adquiridas en su trayectoria migratoria; esto podría dar como resultado que el migrante decida regresar a una localidad, estado o región distinto del que salió cuando migró a Estados Unidos. La evidencia en México sugiere que la propia diversificación que ha experimentado tanto la migración de este país hacia Estados Unidos (origen, sexo, calificación, destino, edad), como la que realizan los mexicanos de manera interna en aquel país, ha favorecido que, al retorno, los migrantes traigan consigo diversos activos (ahorros, habilidades, oficios y conocimientos) (Salas, 2011) que sirven como referencia al momento de decidir a qué lugar arribar a su regreso; incluso la experiencia de generar formas de mantener sus redes sociales a pesar de la distancia les abre la posibilidad de regresar a México sin que necesariamente implique regresar a su localidad de origen inicial, el haber sobrevivido, de una u otra forma, en las urbes norteamericanas puede ser el propulsor para que, a pesar de provenir de comunidades rurales o semiurbanas, al regresar se inserten en zonas urbanas, o incluso escalar de una zona urbana a una metrópoli de mayores dimensiones.

A partir de esta reflexión básica de la migración y el retorno, surge la necesidad de indagar sobre las cifras clave que ponen de manifiesto el fenómeno en el contexto social y le dan visibilidad, aquí encontramos que, de acuerdo a datos de la ENADID 2014, se reportan 264 mil migrantes de retorno, mientras que la EMIF 2014 registró 724 mil eventos de retorno. Por su lado, la Immigration and Customs Enforcement (ICE), en su reporte para el año fiscal 2014, registró 177 mil deportaciones de inmigrantes mexicanos ilegales y de aquellos que incurrieron en alguna actividad delictiva; por su parte, la Secretaría de Gobernación, en su tercer informe de labores 2014-2015 (del 1 de septiembre de 2014 al 30 de junio de 2015), 
reportó que los nacionales repatriados por el gobierno de Estados Unidos ascendió a 174 mil 873 eventos de repatriación, además de registrar nueve mil 842 menores de edad repatriados por Estados Unidos, de los cuales, 8090 eran no-acompañados. Estas cifras hacen evidente que la magnitud del número de eventos y personas reportadas en un año es un evento importante a considerar en los estudios migratorios. En México, por ejemplo, la ida y vuelta, a, y desde Estados Unidos han sido la constante en su devenir, y sus consecuencias no han sido valuadas en su dimensión (Guzmán, 2014).

Es importante poner estos elementos en relieve, ya que no solo dan cuenta del impacto de las leyes migratorias en el comportamiento del retorno y la circularidad migratoria, o del efecto de la crisis económica en la balanza costo-beneficio que enfrentan los migrantes entre quedarse o regresar, sino también son sujetos de una crisis humanitaria, como lo fue el incremento de menores migrantes no acompañados que fueron detenidos por las autoridades fronterizas, el cual se dio a partir del rumor de la posibilidad de alcanzar la ciudadanía estadunidense mediante una solicitud de asilo, trayendo como efecto dominó la atracción de menores migrantes, cuyo principal objetivo no fue precisamente reunirse con sus familiares, sino buscar trabajo, escapar del contexto violento existente en sus lugares de origen.

No obstante, existen diversos factores que influyen en el incremento de la migración de menores: el aumento de la migración femenina, un mayor índice de abandono de los hombres migrantes que interrumpen la comunicación con sus familias (esposas e hijos) y el crecimiento de las remesas (Bustamante, en Valdéz Gardea, 2007), el aumento de la violencia en sus lugares de origen, la escasez de opciones para poder acceder a una mejor calidad de vida, la pobreza y marginalidad, entre otros. Para López (2005), la principal forma de migración de los menores es la familiar, ya sea que viajen con alguno de los padres, o bien, que alguno de estos, o ambos, ya esté en Estados Unidos y estos niños sean "mandados a traer".

Para dar visibilidad a este fenómeno, encontramos que, para 2014, los menores migrantes detenidos por la patrulla fronteriza norteamericana fueron 61 mil, cuando en 2009 tan solo alcanzaban 19 mil (Figura 1). 
Migración internacional de retorno de Estados Unidos a México en un contexto / M. S. MONTOYA ORTIZ et al.

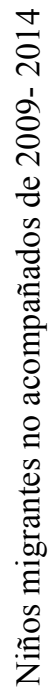
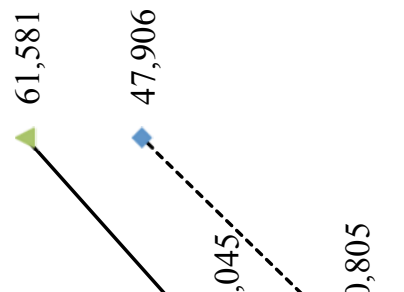

i̊

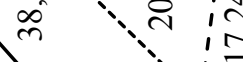

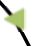
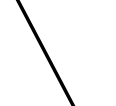

$\int \frac{1}{2}+\frac{1}{11}$

$£$ ले:
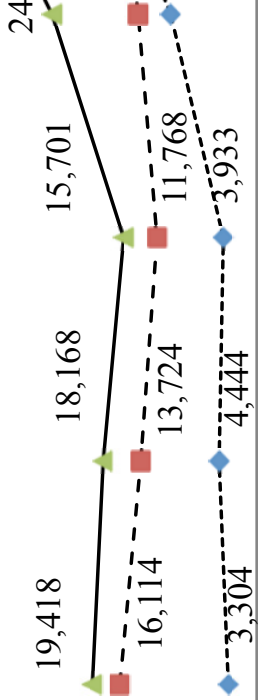

กิ 1 i

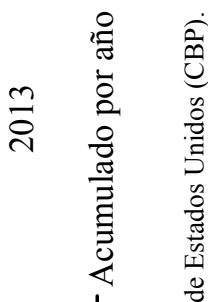

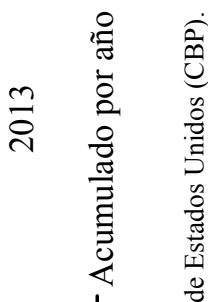

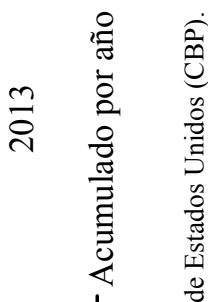

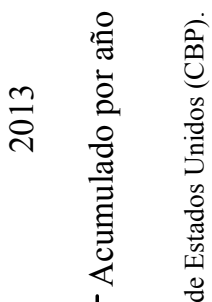

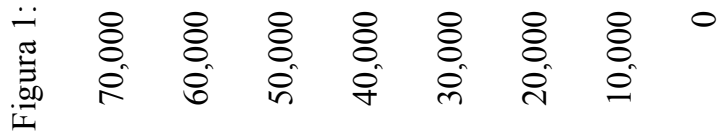

ষ্ণ

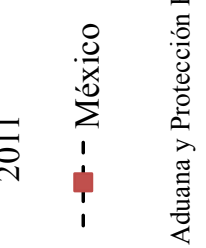

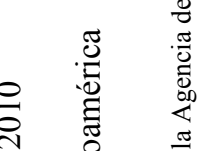

규

$\bigcup_{i}^{0} \quad$ 章

\&

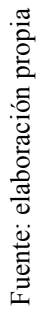


Si consideramos que los menores son doblemente vulnerables, una, por ser menores y otra, por ser migrantes, nos encontramos ante un escenario preocupante que, hasta la fecha, carece de políticas públicas eficaces y adecuadas por parte de ambos gobiernos, el mexicano y el norteamericano, y por otro la invisibilidad que se le ha dado al fenómeno, pues si bien tuvo un amplia cobertura en el año 2014, este fenómeno ya venía ocurriendo desde años anteriores y que aún persiste.

\section{Nuevos Retos en política de MigRación de RETORNo dE MEXICANOS PROCEDENTES de EsTados Unidos}

Hablar de los antecedentes de la política migratoria de México-Estados Unidos es amplio, por lo que solamente se abordará la política de los últimos tres sexenios, que son los que han presentado un mayor impacto en termino de flujos de retorno, y son los que comprenden la base de datos principal empleada en este documento, que es la ENADID 2009 y la ENADID 2014, las cuales abarcan los periodos de 2004 a 2009 y de 2009 a 2014 respectivamente.

Comenzaremos por el periodo de la presidencia de Vicente Fox, quien en 2001 logró dar inicio a negociaciones entre México-Estados Unidos con miras a generar un sistema migratorio que permitiera un flujo ordenado, con la propuesta conocida como the whole enchilada (la "enchilada completa", o el "TLCAN Plus". Durante esta negociación, el retorno de los mexicanos no fue uno de los temas a tratar, en ese momento el retorno no era visto como un punto prioritario, pues, hasta entonces, ambos gobiernos veían el retorno como espontaneo, no problemático y no candidato a la acción de políticas públicas (Alba, 2013). Bueno, más bien, parecía negarse al hecho que desde el gobierno de Bush las deportaciones internas se estaban incrementado. Pero, después de los atentados del 11 de septiembre de 2001, se dio carpetazo a las negociaciones.

En la presidencia de Fox, también fue creada la Oficina Presidencial de Mexicanos en el Exterior (OPME), que tenía como objetivo promover las relaciones comerciales y de inversión mexicana en Estados Unidos, trabajando en estrecha colaboración con la comunidad mexicano-americana. Para 2003 el Programa de Atención a la Comunidad Mexicana en el Exterior (PCME) y OPME se fusionaron creando el Instituto para los Mexicanos en el Exterior (IME), organismo que actualmente es responsable de los programas y fondos en materia migratoria como el 3x1 y 4x1 (Castillo, 2010). 
Si bien, de manera explícita, en el sexenio de Fox no se aborda el retorno, sí se crea el IME, que hoy es el gestor de los programas que pretenden enfocarse a esta población, cuestión que debería haberse abordado por Fox en 2001, que fue uno de los años con mayor flujo de migrantes de retorno, pues el atentado terrorista no solo tocó las fibras de los nativos estadounidenses, sino también de los inmigrantes.

En cuanto al sexenio de Felipe Calderón, Castillo (2010) describe que dentro del Plan Nacional de Desarrollo (PND) 2007-2011, las cuestiones migratorias se ubicaron en el apartado "Mexicanos en el Exterior y Migración", desde donde se plantearon dos objetivos principales, que son proteger y promover activamente los derechos de los mexicanos en el exterior y construir una nueva cultura de la migración.

Aunque no es un sexenio caracterizado por la proactividad en materia migratoria en general, sí fue una etapa anecdótica; Durand (2012) describe que Calderón definió la política migratoria en el sentido de desmigratizar la relación bilateral. Calderón marcaba su raya con el gobierno de Fox, que había insistido de manera recurrente en el tema. Otro elemento importante es un doble discurso del candidato, y luego presidente, panista. En sus mítines acuñó una frase que iba a repetir hasta el cansancio y a voz en cuello: "Yo le digo a los americanos que no gasten tontamente su dinero construyendo un muro, que de todas maneras lo vamos a brincar".

Durand (2012) comenta como, curiosamente, luego de ir a la plaza pública, pasó por la embajada estadunidense el 10 de enero de 2006 a justificarse y pedir la comprensión de los vecinos, aduciendo que no podía darse el lujo de perder ningún voto y que ese tema podía ser capitalizado por su oponente. El doblez salió a la luz cuando se publicaron las revelaciones de Wikileaks en el periódico mexicano La Jornada, acto seguido, redacta Durand (2015), Calderón fue a correr al embajador de Estados Unidos, Carlos Pascual, por decir unas cuantas verdades en los cables privados que enviaba a sus superiores y develados por Wikeleaks.

Aunque, lo que más llama la atención en el sexenio de Calderón, en materia de migración de retorno, es que justo esté sexenio se ve marcado por la crisis económica iniciada en Estados Unidos en 2007 y que alcanzó una escala global, motivo por el cual la migración de retorno incrementó y la emigración se redujo; es curioso porque, el entonces presidente, Calderón, en 2012, declaró "la migración de México a Estados Unidos se han reducido a "casi cero" gracias a las oportunidades en materia de empleo, salud y educación que se han generado" (López, 2012), mientras que Jeffrey Passel, demógrafo del Centro Pew Hispanic, sobre el mismo punto, 
diagnostica que entre las causas de la migración cero destacan la crisis económica y del mercado inmobiliario, la dureza de las leyes migratorias aprobadas por varios gobiernos estatales en los dos últimos años, el peligro que suponen los nuevos puntos de cruce en la frontera y que obligan a atravesar el desierto, el aumento de las deportaciones, el descenso de la natalidad en México y las mejores condiciones económicas en este país. En otras palabras, el endurecimiento de la política de inmigración ha hecho que intentar entrar en Estados Unidos para los mexicanos sea más difícil, caro y peligroso; además, la débil economía estadounidense ha eliminado el imán que suponía la oferta de empleo; y los cambios demográficos en México han reducido el grupo de inmigrantes potenciales" (Pereda, 2012). Así que, de nuevo, el teje y maneje de la política mexicana se queda en el discurso y no pasa a la acción de proyectos que atendieran el retorno de los mexicanos en la etapa de crisis económica iniciada en 2007.

Quizá las posturas políticas de Fox y Calderón no fueron las más eficaces, pero al menos algo se dijo, en contraste nos encontramos con una postura pasiva por parte del presidente Enrique Peña Nieto, a lo que Durand (2015) nombra como "política sin estridencias", es decir, una postura silenciosa, de bajo perfil para evitar molestar al vecino Estados Unidos, una política de conciliación, por no decir sumisa. Esta política ha causado fuertes críticas, pues después de que fuera elegido Donald Trump como presidente de Estados Unidos, que hizo la promesa de campaña para la construcción de un muro fronterizo bajo el lema "build the wall", y la promesa de incrementar las deportaciones de inmigrantes irregulares, con un especial énfasis en los inmigrantes mexicanos, solo se ha hablado de una crisis política entre ambas naciones, pero no de una acción política o un programa social que atienda el fenómeno. Si bien durante el sexenio de Peña Nieto se crearon los programas "Somos Mexicanos", "Construye en Tu Tierra" y "Puertas Abiertas", no se tiene una postura firme ni claridad sobre la línea en materia migratoria, pero Trump sí la tiene, y es aplicar con rigor las leyes inmigratorias e incrementar las deportaciones.

Trump asumió su cargo como el presidente número 45 el 20 de enero de 2017, y en menos de un mes de su gestión, al 11 de febrero de 2017, la Agencia de Inmigración y Aduanas (ICE, por sus siglas en inglés) confirma las primeras redadas de la era "Trump", que han tenido lugar tanto en viviendas como lugares de trabajo de Atlanta, Chicago, Nueva York, Los Ángeles, Carolina del Norte y Carolina del Sur. El ICE ha descrito las redadas donde fueron detenidos cientos de inmigrantes, como "un incremento" en la aplicación de las leyes vigentes (Pereda, 2017). 
Esto no es algo nuevo, las deportaciones de migrantes desde el interior Estados Unidos se han ido incrementando. El principal programa encargado de removals o deportaciones internas es el Enforcement and Removal Operations (ERO) a cargo del ICE, que para el año 2015, reportó 118 mil aprensiones, por lo que queda esperar el reporte de 2018 para ver cómo se comporta esta cifra, la cual se espera que incremente.

En Estados Unidos se puede observar, desde hace varios años, un país divido en materia de política migratoria, por ejemplo, cuando Barack Obama, en 2012, decretó la Acción Diferida para los Llegados en la Infancia o Deferred Action for Childhood Arrivals (DACA, por sus siglas en inglés), cuyo objetivo era regularizar la situación migratoria de las personas que llegaron a Estados Unidos antes de cumplir 16 años y estuvieran en el país desde antes del 15 de junio de 2007; menores de 30 años antes de 2012 y sin antecedentes criminales. La administración Obama enfrentó una demanda legal por parte de 26 estados de la Unión que estaban en contra de esa iniciativa del ejecutivo.

Los 26 estados en contra de esta ley fueron: Texas, Alabama, Arizona, Arkansas, Carolina del Norte, Carolina del Sur, Dakota del Norte, Dakota del Sur, Florida, Georgia, Idaho, Indiana, Kansas, Luisiana, Maine, Michigan, Mississippi, Montana, Nebraska, Nevada, Ohio, Oklahoma, Tennessee, Utah, Virginia Occidental, Wisconsin (Murcia, 2016).

Pero también hay estados más flexibles en materia migratoria, Torres (2011) describe algunos casos, como el de Illinois, que aprobó el 30 de mayo una versión local del DREAM Act; Utah, que aprobó, en 2011, un programa de trabajadores temporales que otorga permisos de dos años a inmigrantes indocumentados, a fin de que puedan viajar al estado para residir y laborar legalmente. Junto con el programa de "trabajadores huéspedes", entraron en vigor nuevas atribuciones para la policía estatal, para que esta pueda cuestionar sobre su estatus migratorio a cualquier persona previamente detenida por otro delito.

Esas facultades, sin embargo, fueron temporalmente bloqueadas por un juez federal. Las organizaciones de migrantes creen que permitir a los agentes locales indagar si una persona está en el país de manera legal o no es discriminatorio, pues favorece el establecimiento de perfiles raciales. Recientemente, en enero de 2017, el gobernador de California, Jerry Brown, levantó la voz a favor de los inmigrantes (Marreo, 2017). Por su parte Nueva York, Washington y Boston también han dado muestras de apoyo mediante masivas protestas contra el veto a inmigrantes de Trump. 
Entre los estados que se pronunciaron en contra del DACA, pero a su vez en 2016 estuvieron solicitando firmas para la aprobación de una ley migratoria para mano de obra inmigrante en las granjas de leche, está Idaho, estado que ha intentado reivindicar su postura migratoria después de la apelación en contra del DACA.

A partir de la compilación periodística mencionada en los últimos párrafos, se presenta el siguiente mapa que muestra el escenario migratorio de Estados Unidos, el cual es un escenario contrastante, y, sin duda, es uno de los principales retos en materia migratoria desde la perspectiva mexicana y estadounidense (Figura 2).

\section{Intensidad migratoria de retorno de Estados Unidos a México DE 2004-2014}

En un primer acercamiento al análisis comparativo acerca del comportamiento de la migración de retorno de acuerdo a los datos presentados por la Encuesta Nacional de la Dinámica Demográfica (ENADID) 2009 y la ENADID 2014, encontramos que la migración de retorno tuvo un decremento en la intensidad migratoria, lo cual representa una variación porcentual de -4.6 por ciento, como lo muestra la Figura 3. Dicha variación porcentual puede hacer referencia a la crisis económica que inició desde el año 2007 y tuvo su punto máximo en 2008, lo que se tradujo en un mayor número de retornos debido a la contracción del mercado de trabajo. La economía estadounidense comenzó a mostrar mejoras a partir del año 2010, lo cual ha permito que el flujo de retorno disminuya y se mantenga estable. A esto habría que sumarle el reforzamiento continuo de las leyes y acciones inmigratorias de Estados Unidos.

Respecto a este último punto, Gandini (et al., 2016) da cuenta de ello: describe que los altos niveles de desempleo en Estados Unidos y el reforzamiento de las medidas implementadas por el gobierno estadounidense para desalentar la migración indocumentada son factores que favorecieron el aumento del flujo de retorno al país.

Además, los periodos de comparación, de 2004 a 2009 y de 2009 a 2014, son periodos críticos en materia migratoria México-Estados Unidos, permiten visualizar cómo se ha comportado el regreso de migrantes en un periodo de crisis económica y posterior a ella, a la vez que dan cuenta del impacto de las leyes más recientes en materia de inmigración aprobadas, como la Ley Sensenbrenner, aprobada en diciembre de 2005 (Border Protection, Antiterrorism and Illegal Immigration Control Act) y la Ley Arizona SB 1070, aprobada en 2009. 
Figura 2: Estados de Estados Unidos anti-inmigrante y pro-inmigrante

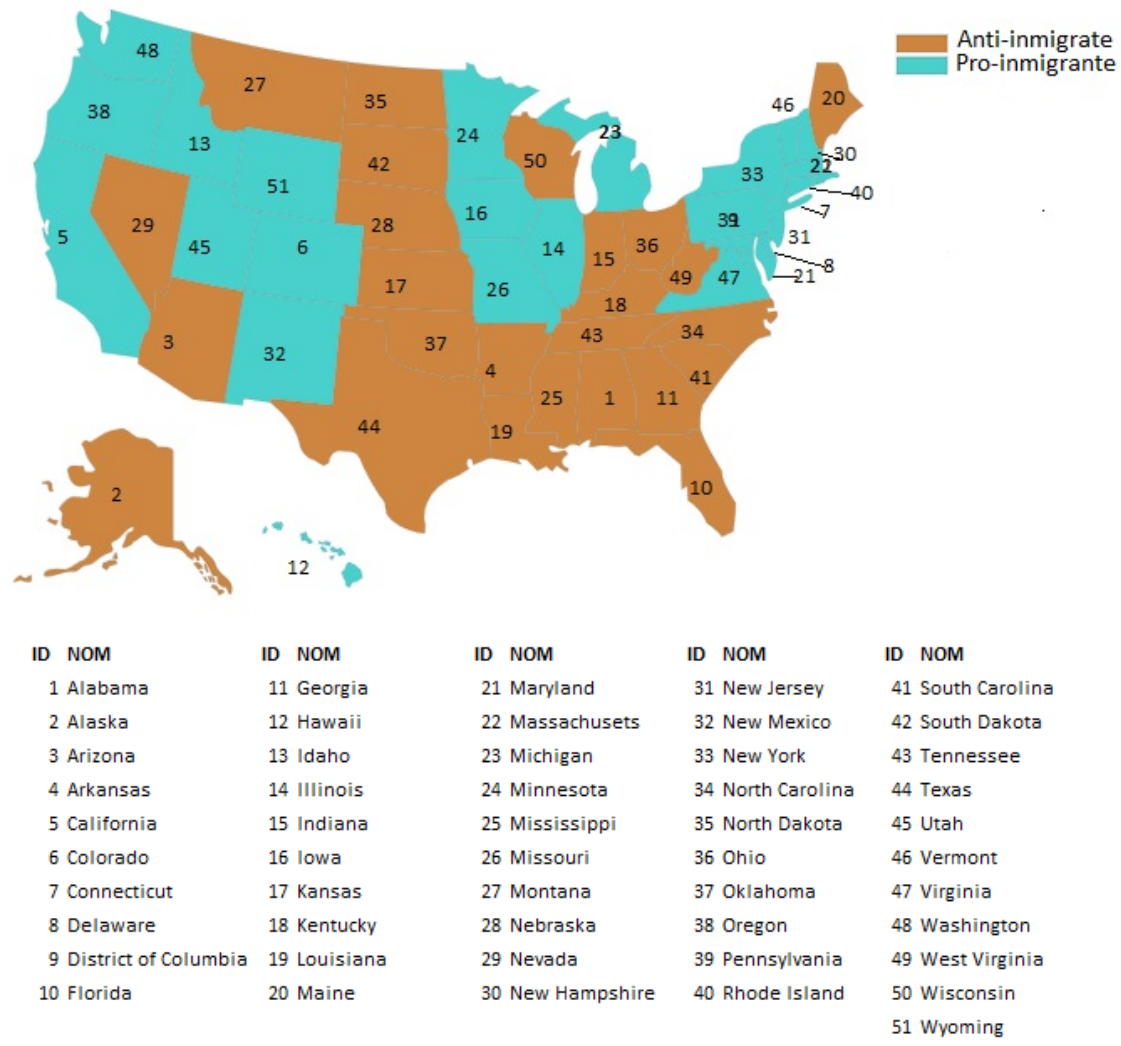

Fuente: elaboración propia a partir de distintas fuentes periodísticas.

Lo cierto es que Estados Unidos se ha limitado a tener una política restrictiva y de expulsión en materia migratoria, y ha evitado generar cualquier sinergia o negociación con el gobierno mexicano incluso, el ICE, con recursos del gobierno estadounidense, es el que financia los centros de detención de inmigrantes en tanto son deportados, con presupuesto para el transporte en que son regresados a sus países de origen incluyendo vuelos especiales para hacer deportaciones a Centroamérica y Sudamérica, y vuelos comerciales en los que deportan a mexicanos. Para el caso de México, la mayoría de los inmigrantes, son devueltos a la frontera y el gobierno mexicano apoya a los inmigrantes deportados a regresar a sus comunidades. 
Figura 3: Volumen de migrantes de retorno para los periodos de 2004 a 2009 y de 2009 a 2014

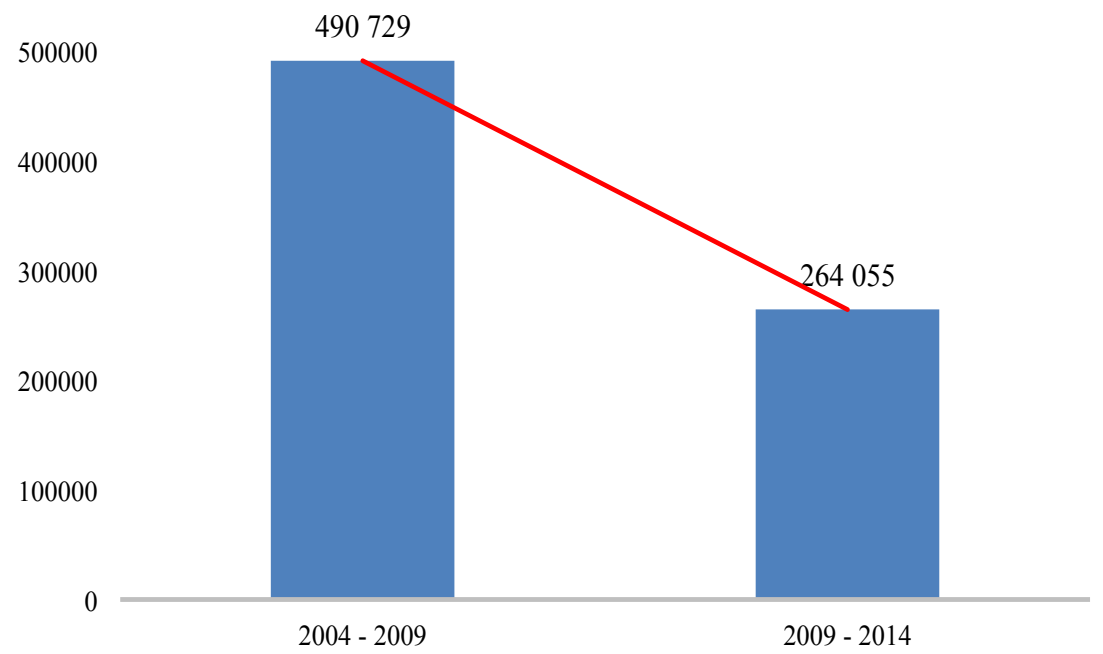

Fuente: elaboración propia con datos de la ENADID 2009 y ENADID 2014.

Tal postura del gobierno estadounidense pudiera ser contradictoria a el objetivo de disminuir la población inmigrante indocumentada en su territorio, pues de generarse alianzas y proyectos productivos, no solo podría motivar el retorno de los inmigrantes sino también evitar una re-emigración.

Por su parte, el Department of Homeland Security (DHS), en su reporte anual, desde 2009 presenta una tabla con cifras de retorno por país, lo que permite constatar la tendencia a la baja en el número de remociones (deportaciones) y de retornos desde 2009 (ver Tabla 1), en donde, para el caso mexicano, se aprecia la constante variación a la baja entre un año y otro.

Otro aspecto a destacar es que, como lo muestra la Figura 4, los retornos tuvieron su punto más alto durante los años de crisis económica, que son 2009 y 2010, a partir del 2011 fueron disminuyendo, en tanto que las remociones muestran un comportamiento contario, a medida que paso la crisis económica las remociones se incrementaron y han mantenido una tendencia a la alta, aquí, entiéndase por remociones, de acuerdo al CBP, como aquellas deportaciones que pasaron por un juicio previo ante inmigración.

Es pertinente señalar que el CBP solo cuenta con cifras por país de los retornos a partir de 2009, por tal motivo no se pudo hacer la comparación para el caso mexicano considerando desde 2004 como lo propuesto para 
este análisis que abarca de 2004 a 2014. Una vez aclarado este punto, tenemos que la DHS sí tiene datos por país de la deportaciones o remociones (removals), aquí se puede encontrar que el comportamiento es irregular, es decir, en algunos años las deportaciones han disminuido y en otros se han incrementado, lo cierto es que la Tabla 2 permite apreciar que para el 2004 el total de remociones era de 176 mil y para 2015 de 242 mil.

Tabla 1: Mexicanos removidos o deportados y retornos: de 2009 a 2016

\begin{tabular}{lrcrr}
\hline Año & Remociones & Retornos & Total & $\begin{array}{r}\text { Variación } \\
(\%)^{*}\end{array}$ \\
\hline 2009 & 276595 & 468722 & 745317 & \\
2010 & 272629 & 353892 & 626521 & -16 \\
2011 & 286972 & 205195 & 492167 & -21 \\
2012 & 301676 & 131818 & 433494 & -12 \\
2013 & 309807 & 88236 & 398043 & -8 \\
2014 & 267649 & 72331 & 339980 & -15 \\
2015 & 235087 & 40528 & 275615 & -19 \\
2016 & 245306 & 37190 & 282496 & 2 \\
\hline * La variación porcentual es calculada sobre el total (deportaciones más retornos) y es respecto \\
al año anterior. \\
Fuente: elaboración propia con datos de la Agencia de Aduana y Protección.
\end{tabular}

Por lo que, finalmente, a pesar de las significativas altas y bajas de las variaciones porcentuales; lo cierto es que las deportaciones de mexicanos se han incrementado en la última década. También se puede evidenciar el efecto de la crisis económica, pues las deportaciones comenzaron su repunte de manera coincidente con el inicio de la crisis en 2007, alcanzando la cifra de remociones más altas en el año 2013, con más de 300 mil deportaciones.

Después de 2013 las remociones tienen una ligera caída, pero el 2016 deja ver que la tendencia incremental volverá, como se observa en la Figura 5, que también muestra de manera visual como el incremento en las remociones no son algo nuevo, han venido en aumento de manera constante desde 2004. 
Figura 4: Comportamiento de los retornos y remociones de mexicanos: de 2009 a 2016

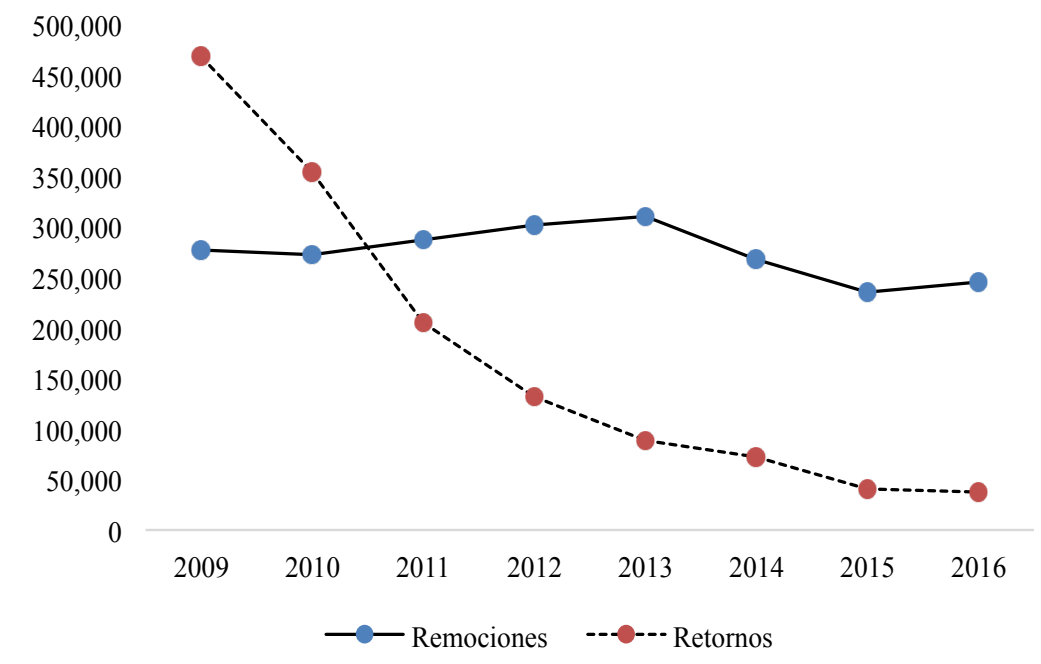

Fuente: elaboración propia con datos de la Agencia de Aduana y Protección Fronteriza de Estados Unidos (CBP).

Tabla 2: Mexicanos removidos o deportados de Estados Unidos: 2004 a 2016

\begin{tabular}{lrr}
\hline Año & Remociones & Variación (\%) \\
\hline 2004 & 175865 & \\
2005 & 169031 & -0.04 \\
2006 & 186726 & 0.10 \\
2007 & 208996 & 0.12 \\
2008 & 247263 & 0.18 \\
2009 & 276595 & 0.12 \\
2010 & 272629 & -0.01 \\
2011 & 286972 & 0.05 \\
2012 & 301676 & 0.05 \\
2013 & 309,807 & 0.03 \\
2014 & 267649 & -0.14 \\
2015 & 235087 & -0.12 \\
2016 & 245306 & 0.04 \\
\hline
\end{tabular}

Fuente: elaboración propia con datos de la Agencia de Aduana y Protección Fronteriza de Estados Unidos (CBP). 
Figura 5: Comportamiento de las remociones de mexicanos de 2004 a 2016

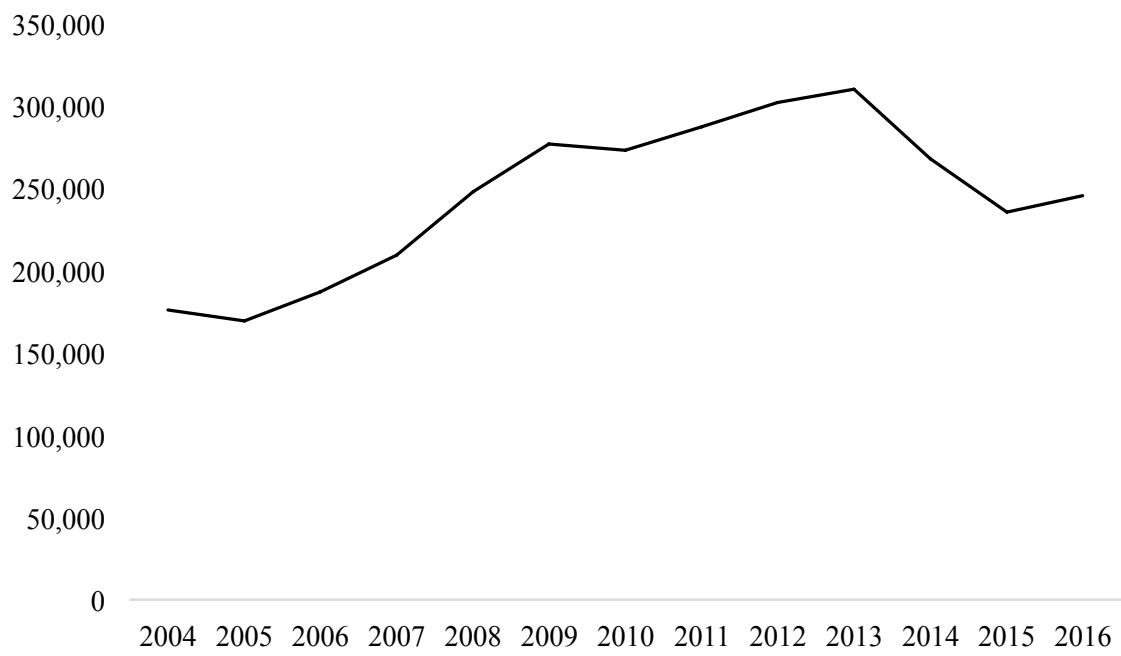

Fuente: elaboración propia con datos del U.S. Department of Homeland Security.

En concreto, los retornos disminuyen mientras las deportaciones incrementan; no obstante, la sumatoria de ambas demuestra una tendencia descendente, por otro lado, las cifras, tanto de la ENADID (2009 y 2014), como del DHS, confirman lo que Jorge Durand (2006) advierte respecto a la pérdida de la circularidad migratoria: los retornos voluntarios disminuyen y los regresos, más bien, se dan de manera involuntaria, es decir que aumentan las deportaciones. Otro aspecto a destacar es que las cifras en números absolutos de la ENADID difieren con lo reportado por el DHS, es importante mencionar que miden cosas diferentes; no solo por aspectos metodológicos, sino porque la ENADID registra a aquellos que ya se encuentran viviendo en México, es decir, instalados en los hogares, mientras que el DHS mide los registros de los que han salido, pero el hecho de que hayan salido de territorio americano no garantiza que necesariamente hayan regresado a México de manera permanente; quizá el migrante ha decidido volver a regresar a territorio americano en lugar de permanecer en México, además, está el problema de los subregistros y el tamaño de la muestra de la ENADID.

Aunado a este escenario de vulnerabilidad, el ahora presidente de Estados Unidos, Donald Trump, tiene una fuerte postura anti-inmigrante, principalmente hacia los mexicanos, y ha prometido frenar la inmigración 
levantando un muro en la frontera México-Estados Unidos, poniendo en la mesa la pregunta "¿Incrementará el retorno de migrantes mexicanos durante la presidencia de Trump?", la historia está por escribirse, pero el panorama no es alentador.

A pesar, de que la meta de Trump es ambiciosa, hay varios factores a considerar, como: la presión internacional sobre la actitud de Trump frente a los inmigrantes, la organización de los propios inmigrantes para defenderse y lo más relevante la cantidad de recursos monetarios humanos y de infraestructura que esto implicaría y correría a cargo de los contribuyentes estadounidenses, cuando en la agenda política se encuentran otros temas que atender como al apertura de empleos de jóvenes, seguridad nacional, salud pública, educación, entre otros. Así que el panorama es gris, el recrudecimiento de las acciones antinmigrantes desalentador, pero no es una causa perdida, y aunque incremento el número de deportaciones difícilmente podrá erradicar la inmigración ilegal o alcanzar a deporta a 11 millones de inmigrantes.

\section{ConClusiones}

A modo de reflexión final respecto a las políticas públicas del migrante de retorno, se puede retomar lo que Castles y Miler (2004) comentan respecto a la migración internacional, diciendo que el saldo general que se deja ver es ambiguo, pues, si bien es importante lo que hacen los diferentes gobiernos, aún se tienen políticas y estrategias migratorias poco claras y efectivas. Ahora bien, para el caso mexicano, el reto en materia migratoria es fuerte, pues la postura pasiva no puede prolongarse, urgen acciones para defender a los connacionales y la soberanía nacional. En materia de migración de retorno, se necesita un programa a largo plazo, al cual se le dé seguimiento y no se le deje en el olvido como ha ocurrido con el Programa de Retorno Humano de 2007. Si durante el mandato de Trump se espera que incrementen las deportaciones, tanto en la frontera como al interior, ¿cómo se va a atender a estos migrantes de retorno?, y ¿qué apoyos se les dará a aquellos que, por el reforzamiento de las leyes inmigratorias y ante un escenario xenófobo, decidan regresar? Estos son los nuevos retos en política de migración de retorno de mexicanos procedentes de Estados Unidos.

Aunque esta postura anti-inmigrante no es garantía de que funcionarán las políticas de Trump, se han incrementado las deportaciones; pero esto no necesariamente se traduce en un mayor número de migrantes retornados o en un incentivo del regreso a México, más bien se ha traducido en la generación de estrategias de sobrevivencia que les permita a los inmigran- 
tes permanecer en ese país, dando continuidad al rompimiento de la circularidad migratoria, expuesto por Jorge Durand (2006), e incluso que se diluyan las redes y lazos sociales que los unen a México. En este sentido, debería de generarse una política migratoria binacional que funcione para ambos países, pues lo cierto es que la migración hacia Estados Unidos puede disminuir, pero no desaparecer; y la pérdida de la circularidad migratoria potencialmente incrementaría la población migrante indocumentada en Estados Unidos, lo cual no es el resultado deseado por dicho gobierno. Ya que, finalmente, el migrante que tiene como objetivo pasar la frontera, la va a pasar; porque la necesidad humana es más grande que una frontera o una policía migratoria. Cuando Trump en su campaña política gritaba build the wall, en las redes sociales cibernéticas circulaban imágenes contestatarias que, de manera irónica, decían "Trump quiere construir muros, pero no sabe que somos expertos en túneles (haciendo referencia a la fuga a través de un túnel del conocido narcotraficante mexicano, "El Chapo Guzmán"). Además de que los reflectores internacionales sin duda serán una presión para evitar que el actual presidente Trump continúe con esta tónica, tal es el caso de las recientes protestas en Londres, Inglaterra, en enero de 2017, bajo la consigna "Construyamos puentes no muros" (Bridges not walls).

\section{REFERENCIAS BIBLIOGRÁFICAS}

Anguiano, María Eugenia, Cruz, Rodolfo y Garbey, Rosa María, 2013, "Migración internacional de retorno: trayectorias y reinserción laboral de emigrantes veracruzanos", en Papeles de Población, núm. 61, Nueva época, julio-septiembre, México.

Bustamante, Jorge, 1997, El marco teórico-metodológico de la "circularidad migratoria": su validación empírica. México.

Bustamante, Jorge A. 1998, "Migración de México a Estados Unidos: un enfoque sociológico", en Bustamante, Jorge A. y Tuirán, Rodolfo (coords.), La migración laboral mexicana a Estados Unidos, El Colegio de la Frontera Norte, Tijuana.

Castillo, Edgar, 2010, La evolución de la política migratoria mexicana: "de la limitación a la participación activa", Universidad Complutense de Madrid.

Castles, Stephen y Miller, Mark, 2004, La era de la migración. Movimientos internacionales de población en el mundo moderno, Colección América Latina y el Nuevo Orden Mundial. México: Miguel Ángel Porrua, UAZ, Cámara de Diputados LIX Legislatura, Fundación Colosio, Secretaría de Gobernación, Instituto Nacional de Migración, México. 
CEPAL, 2005, Desplazamientos: riesgos y oportunidades de buscar nuevos rumbos. Temas de Población y Desarrollo, Comisión Económica para América Latina y el Caribe.

COLEF, 1995, Encuesta sobre Migración en la Frontera Norte de México EMIF NORTE, disponible en http://www.colef.mx

COLEF, 1999-2013, Encuesta sobre Migración en la Frontera Norte de México EMIF NORTE, disponible en http://www.colef.mx

Cruz-Piñeiro, Rodolfo; Ruiz-Ochoa, Wilfrido, 2010, "Migración calificada de mexicanos a Estados Unidos mediante visado preferencial", en Papeles de Población, vol. 16, núm. 66, Universidad Autónoma del Estado de México, Toluca, México.

Durand, Jorge, 2006, "Los inmigrantes también emigran: la migración de retorno como corolario del proceso", en Revista Interdisciplinar da Mobilidade Humana (REMHU), año 14, núms. 26-27, Brasil.

Durand, Jorge, 2012, “Origen y fin de la migración”, en La Jornada, México.

Durand, Jorge, 2015, "Una política sin estridencias", en La Jornada, México, disponible en http://www.jornada.unam.mx/2015/08/09/opinion/015a2pol

Gandini, Luciana, Lozano-Ascencio, Fernando y Gaspar, Selene, 2016, El retorno en el nuevo escenario de la migración entre México y Estados Unidos. Consejo Nacional de Población (CONAPO). México.

González, Gabino y Montoya, Jaciel, 2012, Migración mexiquense a Estados Unidos: un análisis interdisciplinario, Universidad Autónoma del Estado de México.

Granados, José Aurelio y Pizarro Hernández, Karina, 2013, "Paso del Norte, qué lejos te vas quedando. Implicaciones de la migración de retorno en México", en Estudios Demográficos y Urbanos, vol. 28, núm. 2, El Colegio de México, México. pp. 469-496.

Guzmán, Lorena, 2014, Estudio regional sobre Políticas Públicas de integración de migrantes en Centroamérica y México. Sin Fronteras IAP, México.

Herrera, Luis, González Iza, Daniela y Rocha Jiménez, Teresita, 2012, Las políticas públicas ante los retos de la migración mexicana a Estados Unidos: La política migratoria en el nuevo escenario político en Estados Unidos, CONAPO, México.

INEGI, 2010, Encuesta Nacional de la Dinámica Demográfica ENADID 2009, Aguascalientes.

INEGI, 2015, Encuesta Nacional de la Dinámica Demográfica ENADID 2014, Aguascalientes.

INEGI, 2016, Encuesta Nacional de la Dinámica Demográfica ENADID 2015, Aguascalientes.

ICE, 2016, Enforcement and Removal Operations, Immigration and Customs Enforcement. Washington, D.C. Disponible en https://www.ice.gov/ 
López Castro, Gustavo, 2005, Niños, socialización y migración a EstadosUnidosen Michoacán, Woodrow Wilson School of Public and International Affairs, Center for Migration and Development, Working Papers, núm. 361, Princeton University, Princeton.

López, Gustavo, 2012, Los migrantes de retorno y su reinserción a la comunidad, disponible en http://reformacom.typepad.com/otra_tierra/2012/07/los-migrantesde-retorno-y-su-reinserci\%C3\%B3n-a-la-comunidad.html

Massey, Douglas; Pren, Karen y Durand, Jorge, 2009, "Nuevos escenarios de la migración México-Estados Unidos. Las consecuencias de la guerra antiinmigrante", en Papeles de Población, vol. 15, núm. 61, Universidad Autónoma del Estado de México, Toluca.

Marreo, Pilar, 2017, “Gobernador Jerry Brown de California ataca a Trump y defiende a los inmigrantes", en La opinión, Washington, USA, disponible en http:// laopinion.com/2017/01/24/gobernador-jerry-brown-de-california-ataca-a-trumpy-defiende-a-los-inmigrantes/

Murcia, Diego, 2016, "Llega 2016 con incertidumbre para millones de inmigrantes", en El Diario de El Paso, disponible en http://diario.mx/El_Paso/2016-0101_c10aac9b/llega-2016-con-incertidumbre-para-millones-de-inmigrantes/

OIM-INM, 2013, "Perfil socioeconómico y de inserción laboral de los migrantes mexicanos de retorno: análisis comparativo entre 2005-2007 y 2008-2012", Seminario Internacional sobre Migración de Retorno, mayo 2013, Fundación BBVA Bancomer, disponible en http://www.cisan.unam.mx/migracionRetorno/Presentacion_MigRet_UNAM.pdf

Pereda, Cristina, 2012, "El flujo neto de inmigrantes de México a Estados Unidos es por primera vez cero" en El País, disponible en http://internacional.elpais.com/ internacional/2012/04/24/actualidad/1335225354_592757.html

Pereda, Cristina, 2017, "Detenidos cientos de inmigrantes en nuevas redadas en seis Estados de Estados Unidos", en El País, disponible en http://internacional. elpais.com/internacional/2017/02/11/estados_unidos/1486787904_907116.html

Pew Hispanic Center, 2017, Hispanic Trends, disponible en http://www.pewhispanic.org/

Ramírez, Telésforo y Aguado, Daniel, 2013, "Determinantes de la migración de retorno en México, 2007-2009”, en La Situación Demográfica de México, Consejo Nacional de Población (CONAPO), México.

Ravenstein, Ernest, 1885, “The laws of migration", in Journal of the Royal Statistical Society, vol. 48, june. London.

Salas, Renato, 2011, Las aportaciones técnicas y humanas que realizan los migrantes internacionales de retorno en el Estado de México. COFACTOR, México.

Sánchez, Nicolás, 2014, La población en América Latina. El Colegio de México AC, México. 
Secretaría del Zacatecano Migrante, 2017, Fondo de Apoyo al Migrante Zacatecano, disponible en http://transparencia.zacatecas.gob.mx/

Torres, Mauricio, 2011, "El movimiento a favor de los indocumentados cosecha logros en Estados Unidos" en Expansión, disponible en http://expansion.mx/mundo/2011/05/12/leyes-a-favor-de-los-migrantes

Valdéz Gardea, Gloria, 2007, “Geografías rurales olvidadas, menores migrantes en tránsito por el corredor Altar-El Sásabe, expresión moderna del proceso globalizador”, en Méndez Sáinz, Eloy (coord.), Arquitecturas de la globalización, Hermosillo, Universidad de Sonora, Universidad Autónoma de Baja California, Universidad Autónoma de San Luis Potosí, Universidad Autónoma de Nuevo León, Universidad Autónoma de Madrid, Instituto Tecnológico de Estudios Superiores de Monterrey, Mora-Cantúa.

Wong, Rebeca, Resano, Elsa y Martínez, Leticia, 2006, Una constante cambiante: La migración de la población hacia Estados Unidos de Norteamérica, Universidad de Guadalajara, México.

\section{RESUMEN CURRICULAR DE LOS AUTORES}

\section{Merari Stephanie Montoya Ortiz}

Candidata a Doctora en Estudios de Población por la Universidad Autónoma del Estado de Hidalgo. Especializada en tópicos de Migración Interna e Internacional. Estancia de Investigación en la Universidad Complutense de Madrid, España. Publicaciones: 1985, "Evolución de la migración de retorno en México: migrantes procedentes de Estados Unidos en 1995 y de 1999 a 2014", en Papeles de Población, vol. 21 núm. 85, Toluca; 2016, "Índice de migración interna de México, 2014", en Huellas de la Migración, vol. 1, núm. 1, Toluca.

Dirección electrónica: merari.montoya@hotmail.com

\section{José Aurelio Granados Alcantar}

Estudió la licenciatura en economía en la Univeridad Autónoma del Estado de Sinaloa, la Maestría en Demografía en el Colegio de la Frontera Norte y el Doctorado en Planeación Territorial y Desarrollo Regional en la Universidad de Barcelona, España. Trabaja en la Universidad Autónoma del Estado de Hidalgo (UAEH) como profesor investigador desde agosto del año 2000. Desde el 2009 obtuvo el reconocimiento de Profesor Investigador con el Perfil deseable por parte del Programa Nacional de Profesor (PROMEP) y el Sistema Nacional de Investigadores (SNI) le otorgo el nivel I ese mismo año.

Dirección electrónica: joseg@uaeh.edu.mx 
Migración internacional de retorno de Estados Unidos a México en un contexto / M. S. MONTOYA ORTIZ et al.

José Alfredo Jauregui Díaz

Licenciado en Sociología por la Universidad Autónoma de Aguascalientes, Maestro en Demografía por el Colegio de la Frontera Norte y Doctor en Demografía en el Centre d'Estudis Demogràfics de la Universidad Autónoma de Barcelona. En el sector público ha trabajado como asesor en los Consejos Estatales de Población de Quintana Roo y Chiapas. El Dr. Jáuregui es Investigador Nacional Nivel I del Consejo Nacional de Ciencia y Tecnología (CONACYT). Actualmente, es Profesor Investigador en el Instituto de Investigaciones Sociales de la Universidad Autónoma de Nuevo León, México. En el ámbito académico tiene diversas publicaciones. Sus líneas de investigación son migración interna e internacional, dinámica sociodemográfica y desarrollo sustentable.

Dirección electrónica: alfjadi@yahoo.com.mx

Artículo recibido el 19 de septiembre de 2017 y aprobado el 14 de febrero de 2018. 